\title{
Fast and Precise Measurement in the sub-20 nm Size Range using a Scanning Mobility Particle Sizer
}

\author{
Jasmin Tröstl ${ }^{\mathrm{a}}$, Torsten Tritscher ${ }^{\mathrm{b}}$, Oliver F. Bischof ${ }^{\mathrm{b}}$, Hans-Georg Horn ${ }^{\mathrm{b}}$, Thomas \\ Krinke $^{\mathrm{b}}$, Urs Baltensperger ${ }^{\mathrm{a}}$, Martin Gysel ${ }^{\mathrm{a}, *}$ \\ ${ }^{a}$ Laboratory of Atmospheric Chemistry, Paul Scherrer Institute, Villigen, Switzerland \\ ${ }^{b}$ TSI GmbH, Particle Instruments, Aachen, Germany
}

\begin{abstract}
An increased number of studies are focusing on the detection and investigation of nanometer-sized particles. One important tool is the Scanning Mobility Particle Sizer (SMPS). In the case of dynamic processes like nucleation events, the lower detection threshold as well as the measurement time are important system parameters. The limiting factors which determine the accuracy of the SMPS are the high voltage accuracy under dynamic conditions and knowledge of the response function of the detector. Especially the quality of the sizing depends on the accuracy of applied voltages in the differential mobility analyzer. High accuracy of the applied voltage as a function of time during fast scanning is required. Short scan times additionally yield a broadening and smearing of the measured size distribution. Here, the performance of the Nano-SMPS using the new TSI classifier (TSI 3082) in combination with either the nano water condensation particle counter (TSI 3788) or the ultrafine condensation particle counter (TSI 3776) was investigated. The focus of this work is the performance of the system at the lower detection limit as a function of the scan time. Fast scan times as short as three seconds were tested and compared to quasi-stationary measurements. The reproducibility of the aerosol size distribution and number concentration for different distributions and materials was investigated. The tested substances included different proteins (myogobin, ubiquitin, ferritin, albumin), sucrose and polystyrene latex reference particles. The sizing reproducibility and accuracy for all tested scan times was within $3 \%$. This is only possible, by determining the delay time precisely. In addition, the limitations in size resolving power due to smearing and broadening resulting from fast scan times were investigated and quantified. The measured total number concentration was captured with a precision of $\pm 3 \%$ for all tested scan times. The determination of the absolute concentration for this size range was not considered and remains a challenge for future studies, as diffusion losses, charging probability and CPC counting efficiency are critical issues.
\end{abstract}

\section{(C) 2014 Published by Elsevier Ltd.}

Keywords: Nanoparticles, Fast Scanning, Nano Scanning Mobility Particle Sizer (Nano-SMPS)

\section{Introduction}

Atmospheric aerosols impact the global radiation balance and, therefore, play an important role in climate change (Stocker et al., 2013). They also cause adverse health effects (Hoet et al., 2004), particularly ultrafine particles $(<100 \mathrm{~nm})$ that can penetrate deeply within the lungs, and even cross the cell membrane to enter blood and lymph circulation systems, eventually reaching sensitive areas such as heart and bone marrow (Peters et al., 1997). Atmospheric new particle formation events - so-called nucleation events - produce ultrafine particles that grow to cloud

${ }^{*}$ Correspondence to: Martin Gysel, Paul Scherrer Institute, 5232 Villigen PSI, Switzerland. E-mail: martin.gysel@psi.ch, Phone: +41 56 310 $-4168$

This document is the accepted manuscript version of the following article:

Tröst1, J., Tritscher, T., Bischof, O. F., Horn, H. G., Krinke, T., Baltensperger, U., \& Gyse1, M. (2015). Fast and precise measurement in the sub-20nm size range using a scanning mobility particle sizer. Journal of Aerosol Science, 87, 75-87.

https://doi.org/10.1016/j.jaerosci.2015.04.001

This manuscript version is made available under the CC-BY-NC-ND 4.0

1icense http://creativecommons.org/1icenses/by-nc-nd/4.0/ 
condensation nuclei (CCN) and account for up to $45 \%$ of the global CCN in low level clouds (Merikanto et al., 2009). These important effects have stimulated an increasing number of studies that focus on the detection and properties of nanometer-sized particles challenging available characterization methods and techniques to lower limits of detection, to the lowest particle size. Mobility particle size spectrometers are the most common instruments used to size airborne nanoparticles. They consist of a differential mobility analyzer (DMA) (Knutson and Whitby, 1975) that selects aerosol particles of a certain size according to their electrical mobility; the selected particles are then measured by a condensation particle counter (Quant et al., 1992). Originally, different particle sizes were selected by stepping the voltage of the DMA, a process that took several minutes.

In 1990, the concept of scanning the voltage was first introduced by Wang and Flagan (1990). Since then, the scanning mobility particle sizing or SMPS technique has been established as the method of choice due to its rapid measurement principle that speeds up measurements without loss of accuracy and resolution. Nano scanning mobility particle sizers (Nano-SMPS) extend these measurements to the 3-50 nm size range (nano-DMA design by Chen et al., 1998). In spite of the accelerated measurement, the SMPS still has limitations for the measurement of rapidly varying number size distributions, e.g. fast growing nanoparticles during nucleation experiments. With single scans typically lasting $60 \mathrm{~s}$ or more, the transient aerosol size distributions remain difficult to capture, limiting our ability to understand aerosol nucleation and early particle growth processes. Accurate size determination with a high size and time resolution and high counting efficiency with a detection limit down to a few nanometers is, therefore, crucial for field and laboratory experiments focusing on nucleation and growth (Riccobono et al., 2014; Kulmala et al., 2012). In addition, direct emission measurements from transient aerosols such as combustion processes and vehicles can benefit from improved time resolution (Zhu et al., 2002). Another potential application is the characterization of engineered nanoparticles, which become more and more important in industry and the environment (Tiede et al., 2008).

Two experimental factors limit the speed with which size distributions can be measured: (1) The ability of the high voltage power supply to accurately apply and rapidly scan the full voltage range; and (2) the response of the CPC detector. For long scan times, the latter effect is minor, but during fast scanning the number size distribution can be broadened and also skewed compared to the true distribution (Russell et al., 1995). Thus, one approach would be to improve the inversion by accounting for the finite width and asymmetry of the CPC response function to correct for the smearing (Collins et al., 2002; Mamakos et al., 2008). However, the inversion of smeared data is limited by uncertainties in the kernel function and random noise in the measurement. Thus, an improvement of the CPC response function is preferred.

Several studies have tried to decrease the scan time of different measurement systems by improving the instrumentation. In 2005 for instance, Shah and Cocker presented a fast-SMPS with scan times down to $2.5 \mathrm{~s}$ (scan range 5 - $98 \mathrm{~nm}$ ). They used a mixing-type state CPC (Wang et al., 2002) to optimize the CPC response time. However, fast scanning tests for the sub-20 nm range (such as monodispers particles or an intercomparison with a reference scan) were not shown. Alternatively several instruments with very high time resolution (a few seconds or less) are currently available, e.g. the Fast Integrated Mobility Spectrometer (FIMS) (Olfert et al., 2008) or the Engine Exhaust Particle Sizer (EEPS) (Johnson et al., 2004). These instruments also classify the particles according to their electrical mobility. The FIMS uses a charge-coupled device (CCD) camera to detect the particles, however to allow for good counting statistics, not more than 10 size channels are recommended. For the EEPS, the particles are detected as electrical current using a number of electrometers that detect particles in parallel in defined size channels. Thus, it is possible to monitor several size channels simultaneously instead of scanning through a certain diameter range. Due to the finite number of detectors, these instruments have a limited size resolution. Thus, combining lower detection limits, fast scanning, and high size resolution still remains difficult in the sub- $20 \mathrm{~nm}$ size range.

In this work we show that fast and precise scanning is possible without the need to apply complex inversion algorithms by combining recently developed state-of-the-art instrumentation. We characterize the new Nano Scanning Mobility Particle Sizer (Nano-SMPS, TSI 3938). The Nano-SMPS consists of a novel classifier (TSI model 3082) combined with a Nano-DMA (TSI model 3085A) using either the Nano Water-based Condensation Particle Counter (N- WCPC, TSI model 3788) or the butanol based Ultrafine Condensation Particle Counter (UCPC, TSI 3776).The N-WCPC has a very short rise time $(<100 \mathrm{~ms})$ and, thus, a very narrow response function. In addition the high voltage supply has been improved so that it can accurately control the voltage during fast scanning, and can also operate reliably to lower voltages than was possible in earlier instruments. These improvements allow accurate size determination and reduce the scan time without smearing. This article presents the performance and limitations of the new Nano-SMPS for scan times down to $3 \mathrm{~s}$. 


\section{Experimental Methods and Sample Preparation}

\subsection{New Nano Scanning Mobility Particle Sizer}

We used the new Nano Scanning Mobility Particle Sizer (Nano-SMPS, TSI 3938), which is based on a novel Electrostatic Classifier (EC, TSI 3082). The calibration of this classifier fully complies with ISO standard 15900, which provides the methodology for quality control of differential electrical mobility analysis. The EC features several essential improvements over previous designs including: (1) a higher maximum sheath flow rate of $30 \mathrm{~L} \mathrm{~min}^{-1}$ for increased size resolution and less diffusion losses, (2) a precision dynamic high voltage supply with a response time of less than $50 \mathrm{~ms}$ which enables accurate and fast scanning and with improved settings at lower voltages and (3) the option of a dual polarity high voltage supply for the Differential Mobility Analyzer (DMA), such that it can be switched to study oppositely charged particles. The EC uses a strong single sheath flow pump and a mass flow meter to precisely control the closed loop sheath flow. Relative humidity, temperature and absolute pressure are always measured within this sheath flow system. Either a $\mathrm{Kr}-85$ or an X-ray neutralizer can be inserted into the classifier. To achieve aerosol charge equilibrium a soft X-ray charger (TSI model 3088) was installed in this study. The charge equilibrium of particles bigger than $20 \mathrm{~nm}$, can been determined very accurately, but the exact charge equilibrium of sub-20 nm particles still remains a challenge (Lòpez-Yglesias and Flagan, 2013). The operation schematic of the EC is presented in Figure 1.

During all our measurements the EC was configured with a Nano Differential Mobility Analyzer (Nano-DMA, TSI 3085A), described in more detail by Chen et al. (1998). The voltage applied to the DMA for size selection was negative at all times. As a detector we chose the Nano Water-based Condensation Particle Counter (N-WCPC, TSI 3788), as this instrument offers a response time of $250 \mathrm{~ms}$ and less than $100 \mathrm{~ms}$ rise time. This very short rise time improves the resolution for very fast scans. The combination of this very fast N-WCPC with the dynamic high voltage power supply permits very fast scanning with data sampling rates up to $50 \mathrm{~Hz}$. In addition, the N-WCPC provides good counting statistics at low particle number concentration due to its comparatively high sample flow rate through the detection volume of $0.3 \mathrm{~L} \mathrm{~min}^{-1}$. The inlet flow can be set either to 0.6 or $1.5 \mathrm{~L} \mathrm{~min}^{-1}$. Details on the $\mathrm{N}$-WCPC can be found elsewhere (Kupc et al., 2013; Hering et al., 2005; Hering and Stolzenburg, 2005). In some cases (when stated explicitly) the butanol-based Ultrafine CPC (UCPC, TSI 3776) was used. This UCPC has a rise and response time of $160 \mathrm{~ms}$ and $640 \mathrm{~ms}$, respectively and a sample flow rate of $50 \mathrm{~mL} \mathrm{~min}^{-1}$. The Nano-SMPS was operated at a sheath to aerosol flow ratio of $10: 1$, with flow rates of $15 \mathrm{~L} \mathrm{~min}^{-1}$ and $1.5 \mathrm{~L} \mathrm{~min}^{-1}$ (resulting in a maximum size range of $2-65 \mathrm{~nm}$ ) unless stated otherwise.

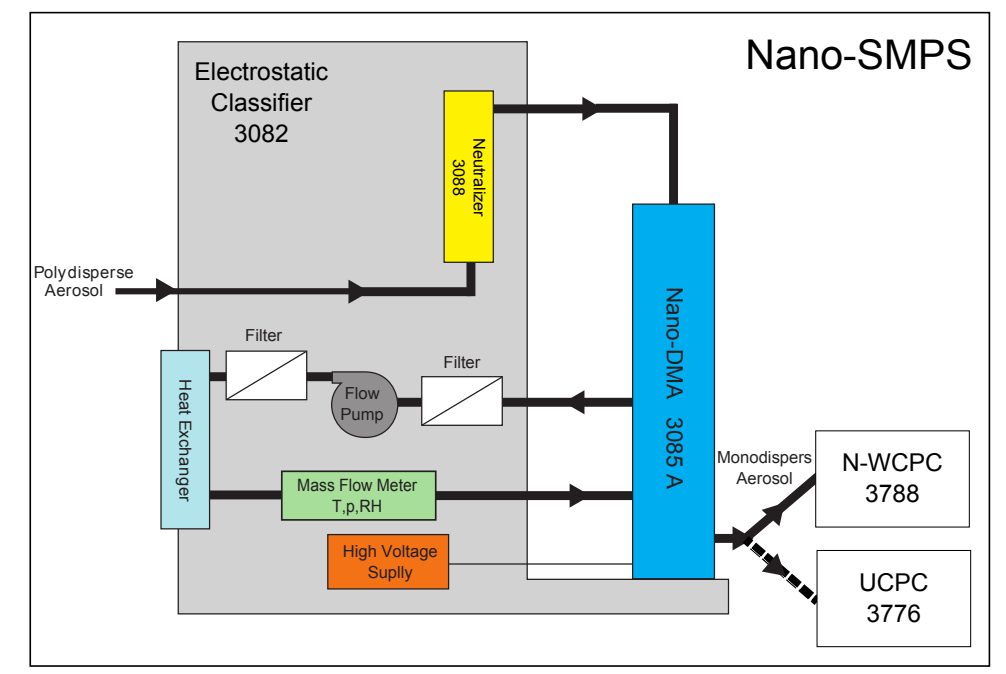

Figure 1. Nano-SMPS. Operation schematic of the new Electrostatic Classifier (EC, TSI 3082), with the new sheath flow pump for increased flow rate and the precision dynamic, the soft-x-ray bipolar aerosol neutralizer (TSI 3088), Nano-DMA 3085A and CPC. Most of the time the water-based N-WCPC was used, in some cases (when stated explicitly) a UCPC 3776 was used. (Color figure available online.) 


\subsection{Setup and Aerosol Generation}

All experiments were performed at standard laboratory conditions and CPC operating temperatures. The different test aerosols used in this study were generated with an Electrospray Aerosol Generator (EAG, TSI 3480). In the EAG, a liquid solution or suspension of a certain electrical conductivity is pushed through a $25 \mu$ m diameter capillary tube. By applying an electric field to the tip of the capillary, droplets of an initial size of $150 \mathrm{~nm}$ are produced. The freshly formed droplets are highly charged. During evaporation inside the EAG chamber, their charge is rapidly reduced using alpha radiation from a Po-210 source $(185 \mathrm{MBq})$, to avoid disintegration of the droplet (Kaufman, 1998). Afterward, an additional X-ray charger was used in the Nano-SMPS to establish charge equilibrium for the fully dried aerosol particles (see section 2.1). Salt or sucrose particles are produced from an aqueous solution, with the sizes of these particles being a function of the concentration of the solution. The EAG generated particles smaller than $50 \mathrm{~nm}$, which are singly charged when in equilibrium state with only a negligible amount of doubly-charged particles. Further details on the EAG can be found elsewhere (Chen et al., 1995; Kaufman, 1998).

Aerosol standards were used in a first approach to quantify the sizing accuracy of the Nano-SMPS. For this purpose, the EAG was directly connected to the Nano-SMPS (Figure 2 Setup A). The output of the EAG was adjusted to $2.5 \mathrm{~L} \mathrm{~min}^{-1}$. Downstream of the EAG, an open outlet with approximately $1 \mathrm{~L} \mathrm{~min}^{-1}$ excess air was installed to allow the Nano-SMPS to operate at $1.5 \mathrm{~L} \mathrm{~min}^{-1}$ aerosol flow. We connected a HEPA filter and a mass flow meter (TSI model 4140) to the open outlet to monitor the excess air.

In a second type of experiments (Figure 2 Setup B), a tandem Nano-DMA setup was applied to further assess the reproducibility and accuracy of the sizing. The output of the EAG was adjusted to $2.5 \mathrm{~L} \mathrm{~min}^{-1}$. The EAG was connected to the first Nano-DMA run with a second EC unit. Between the two DMAs a dilution flow was added, which was controlled by a needle valve and monitored by a mass flow meter. Thus, the sample flow rate through the first Nano-DMA was constant at all times. We used the Nano-DMA to select particles of a defined mobility diameter by choosing the corresponding voltage for $30 \mathrm{~L} \mathrm{~min}^{-1}$ sheath flow rate. To keep the sheath to aerosol flow ratio of 10:1 and to dilute the monodisperse aerosol, $4.5 \mathrm{~L} \mathrm{~min}^{-1}$ of dilution air were added after the first Nano-DMA. A mixing orifice was installed to still provide a uniformly mixed aerosol flow. Afterwards we used a flow splitter to provide the sample flow for the CPC and additional instruments and/or simply a vent for excess air. No data from the additional instruments are included in this study. We used conductive sampling lines that were kept as short as possible to minimize diffusion losses. Sheath, dilution and inlet flows were checked and monitored with mass flow meters and a bubble flow meter (Gilian, Gilibrator-2).

We generated particles of six different compositions in the size range between 2 and $107 \mathrm{~nm}$ diameter using
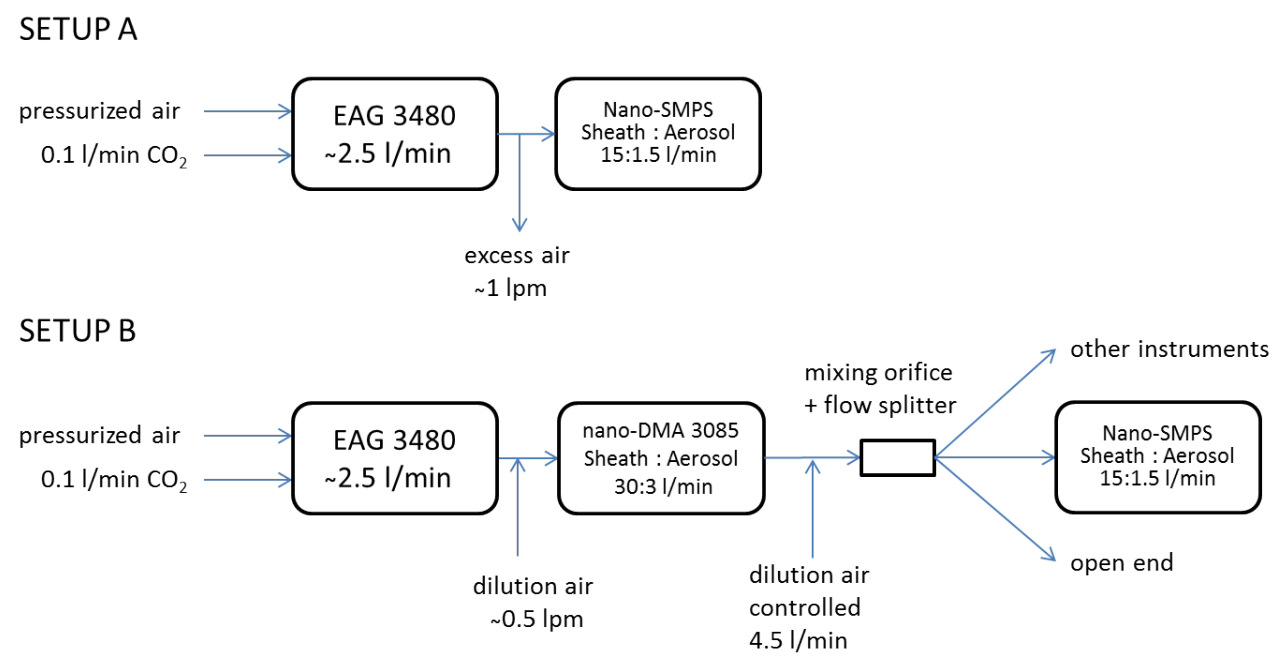

Figure 2. Schematic of the characterization experiment showing two different testing setups. Setup A was used to acquire size distributions of different electrosprayed solutions. Setup B was used to generate monodisperse distribution which were then subsequently measured with the Nano-SMPS. For this a second DMA was needed to select particles of a certain size beforehand. 
the EAG: (1) polystyrene latex (PSL) reference particles as size standard (Duke scientific micro particles), (2) sucrose (ACS, Alfa Aesar), and the proteins (Sigma-Aldrich) (3) ferritin from equine spleen (F4503), (4) albumin from bovine serum (A7638), (5) myoglobin from equine skeletal muscle (M0630), and (6) ubiquitin from bovine erythrocytes (U6253). PSL, sucrose and protein solutions were prepared in a $20 \mathrm{mM}$ ammonium acetate buffer solution with a conductivity of $0.2 \mathrm{~S} \mathrm{~m}^{-1}$ (Chen et al., 1995).

The particle size from sucrose solutions depends on the dilution (Kaufman, 2000), thus, we prepared different ones with concentrations ranging from 1 to $1500 \mathrm{ppm}$ (parts per million). Albumin, myoglobin, ubiquitin and ferritin solutions with approximately $0.26 \mathrm{mg} \mathrm{ml}^{-1}$ were prepared in a $20 \mathrm{mM}$ ammonium acetate buffer solution. We used then this stock solution to prepare several solutions by diluting them by a factor 10 and/or 100 for some experiments. Before installing the samples in the EAG, we put the suspension for at least two minutes in an ultrasonic bath to ensure a good suspension of the proteins. The size of the protein peaks does not depend on the dilution, as they are only suspended and not dissolved. The ratio of mono- and multimers, however, changes with concentration. With higher concentration a bigger fraction of the protein molecules clusters to di- or trimers. Thus, we tested different dilutions to verify the protein sizing as described in section 3.1 .

\subsection{Data Analysis}

Aerosol number size distributions (for brevity from hereon referred to as size distribution) were retrieved from the raw data using the Aerosol Instrument Manager (AIM), Version 10.1. The inversion of the raw data is based on the detailed description by Wang and Flagan (1990). The standard SMPS inversion algorithm that was applied contains the following common assumptions: The size distribution $d N / d \log D_{p}$ is assumed to be constant across the width of the transfer function for all sizes. This directly implies that only the area of the DMA transfer function has an influence on the inverted size distribution, while its width and shape do not. Thus, the idealized triangular DMA transfer function without accounting for diffusion broadening was used to calculate the area, as diffusion broadening does not alter its area. The simplified inversion is virtually exact for broad and smooth size distributions. Quasimonodisperse size distributions, which do not fulfill the above assumption, where investigated in several experiments of this study. The simplified inversion still provides the integrated number concentration correctly, while the width of the size distribution will be slightly overestimated. However, this is irrelevant for the purpose of this study, as only the precision of the measured width in dependence of scan time is of interest. Using a full inversion scheme with accounting for diffusion broadening would only be needed, if the absolute width of measured narrow size distributions was of interest.

The SMPS inversion algorithm also corrects for the size dependent single charge probability, the effects of multiply charged particles (Knobel et al., 2013) and the CPCs counting efficiency across the range of its lower size cut-off. We excluded data points at diameters below the CPCs $50 \%$ counting efficiency cut-off. Specific CPC calibrations would be needed in that range, as uncertainties associated with the exact shape of the cut-off curve in dependence of particle chemical composition become increasingly large. This is outside the scope of this study. For more information on the N-WCPC cut-off curve see Kupc et al. (2013). Diffusion losses within the Nano-SMPS were corrected using the widely accepted effective length approach (Wiedensohler et al., 2012). To obtain accurate size distribution measurements, it is necessary to characterize the diffusion losses in the complete sampling manifold and DMA unit. We applied the literature value for the effective length for the Nano-SMPS, which was already derived from previous studies (Wiedensohler et al., 2012; Chen et al., 1998; Birmili et al., 1997; Reineking and Porstendörfer, 1986) and experimentally validated by TSI. Uncertainties associated with charging probability, CPC cut-off curve and diffusion losses limit the accuracy of measured particle size distributions, particularly in the sub-20 nm size range. However, this does not impair the sizing accuracy nor the precision of number concentrations obtained from repeated measurements with different scan times of the same sample, which were the subjects of this study.

To investigate the performance of the Nano-SMPS for fast scanning measurements, we considered the scan velocity in addition to the scan time, which is the time needed to perform one scan over a predefined size range. The scan time itself does not provide all relevant information as the scanned diameter range can vary and, thus, influence the instrument performance at fixed scan time. The scan velocity $v_{s}$ was determined according to Weingartner et al. (2002):

$$
v_{s}=\frac{d \log _{10}\left(D_{p}\right)}{d t}
$$


A
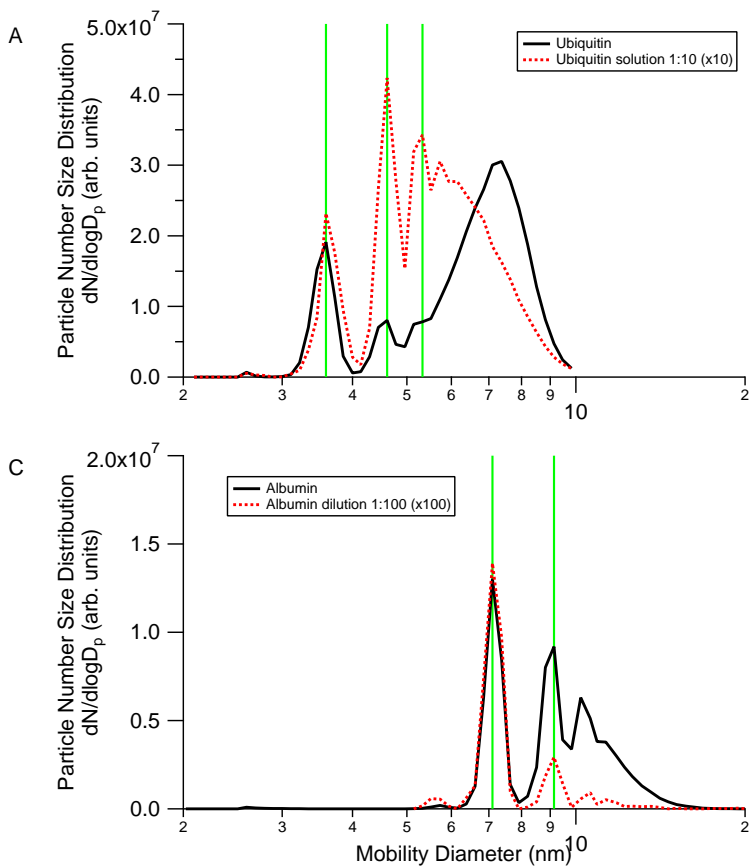

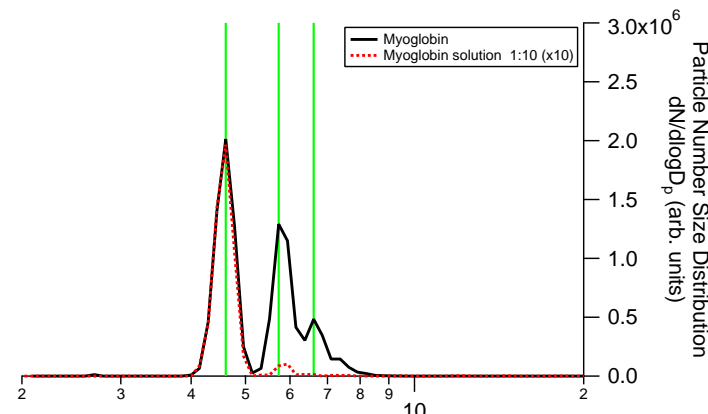

B

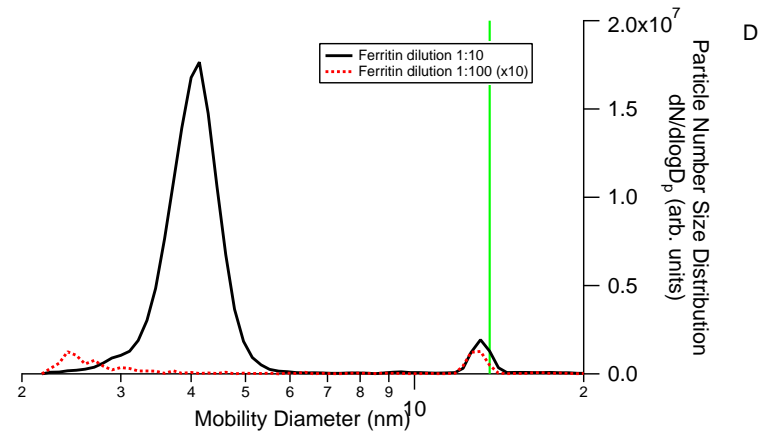

Figure 3. Observed protein peaks. Two different solutions with varying concentration of (A) ubiquitin, (B) myoglobin, (C) albumin and (D) ferritin are displayed. The dashed lines correspond to the diluted distributions which were multiplied with the dilution factor to simplify the comparison. The solid lines correspond to the stock solutions or a weaker solution in case of Ferritin, respectively. The positions of the protein peaks according to (Bacher et al., 2001) are indicated with the vertical lines. (Color figure available online.)

which corresponds to the change in diameter $D_{p}$ per time on a logarithmic scale. A scan velocity of $v_{s}=1 \mathrm{~s}^{-1}$ corresponds to one size decade per second. High scanning velocities can not only lead to smearing effects but can also introduce additional sizing uncertainties corresponding to errors in the delay time, which is the time between DMA monodisperse exit and detection by the CPC. The corresponding error is the following according to Weingartner et al. (2002):

$$
\frac{\Delta D}{D}=10^{v_{s} \Delta t_{d}}-1
$$

whereas $t_{d}$ is the delay time and $\Delta D / D$ the relative error. For fast scanning, e.g. $0.2 \mathrm{~s}^{-1}$, a deviation of $0.1 \mathrm{~s}$ from the correct delay time yields a $5 \%$ error in the sizing. Thus, for fast scans the determination of the delay time is crucial for correct sizing. The delay time was empirically determined and optimized so that the measured particle size became independent of the scan time.

The geometric mean diameter $\left(D_{g}\right)$ was chosen as a representative size of the measured mode. We used two approaches to determine $D_{g}$. For isolated modes, the integral over the mode according to the statistical definition was calculated. For overlapping modes, we fitted a multi-modal lognormal distribution. The width of the mode is expressed as the geometric standard deviation $\left(\sigma_{g}\right)$. It is used to quantify potential smearing effects at fast scan times. $\sigma_{g}$ was determined in the same manner as $D_{g}$, either by integrating over the mode for isolated modes, or by fitting multi-modal lognormal distributions.

\section{Results}

\subsection{Sizing Accuracy}

\subsubsection{Quasi-stationary Tests}

With stationary size distribution measurements, i.e., stepping the voltage, the potential bias from transient effects and finite response times can be avoided. However, such transient effects can also be avoided with very slow, quasi- 

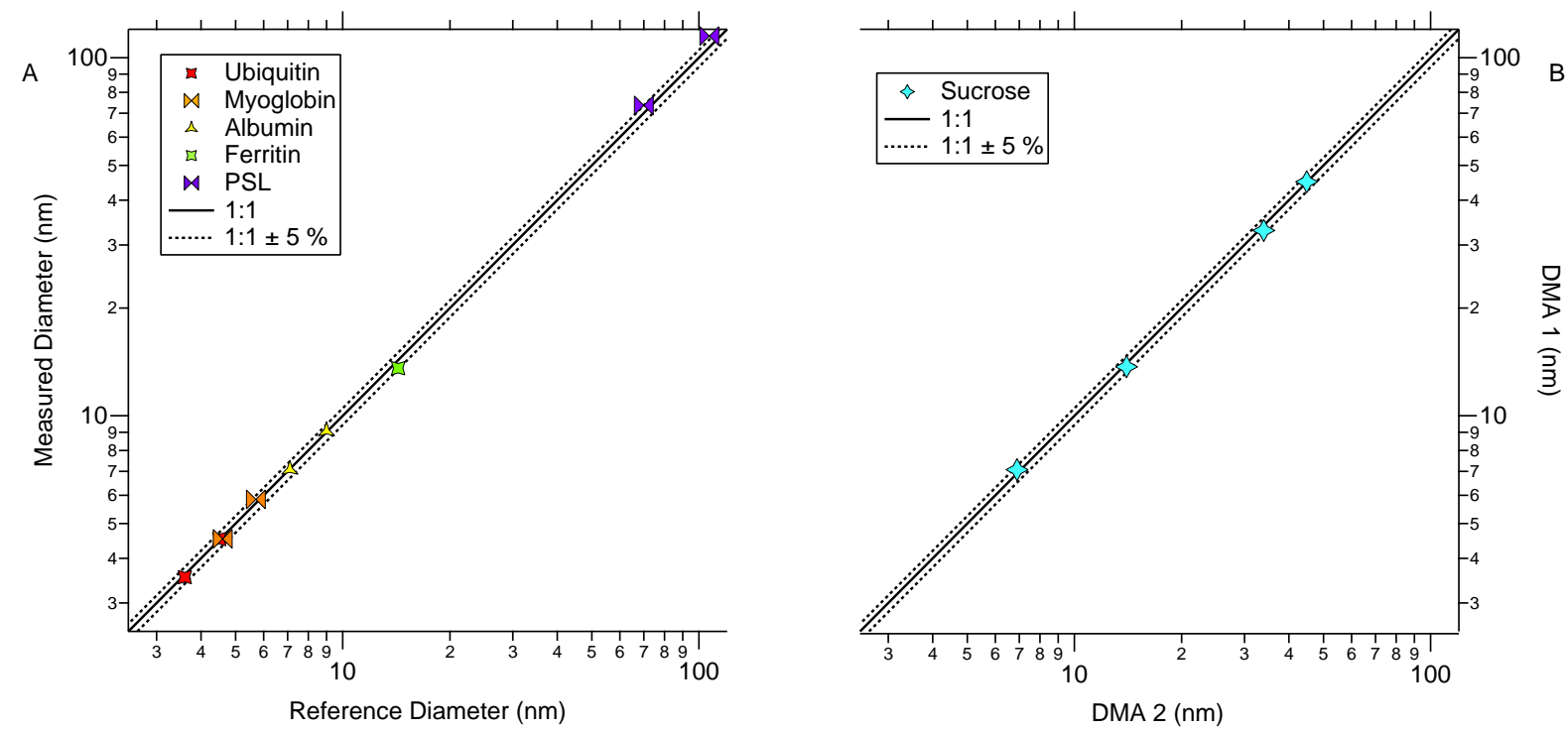

Figure 4. SMPS sizing experiment. The left plot shows the observed $D_{g}$ in comparison to the reference values from literature or manufacturer data. The standard deviation was less than $1 \%$ and is, therefore, not plotted. The right plot shows the comparison of SMPS 1 and SMPS 2 to verify the two DMAs against each other. Also here the standard deviation was less than $1 \%$ and, thus, not plotted. (Color figure available online.)

stationary scans. In order to investigate the sizing accuracy, we tested the Nano-SMPS with substances of a known diameter using the first experimental setup (Figure 2A, Sect. 2.2). Comparing size distributions measured with scan times of $50 \mathrm{~s}$ with those measured with scan times $>100 \mathrm{~s}$, we did not see a detectable difference.

Therefore, $50 \mathrm{~s}$ scans already provide equal results to a stepping approach measurement and are chosen as a reference scan. To investigate diameters below $20 \mathrm{~nm}$, the proteins listed in Table 1 were used. Each protein was tested in two different concentrations, directly using the stock suspension (see Sect. 2.2) and a 10-fold dilution (10 and 100-fold dilution in case of ferritin). Figure 3 shows the measured (single scan) protein size distributions for a scan range from 2 to $65 \mathrm{~nm}$. Measurements of the (relatively) concentrated and dilute suspensions are indicated with solid and dashed lines, respectively. To guide the eye, the vertical lines indicate the mobility diameter of the mono-, di- and trimers (if applicable) reported by Bacher et al. (2001). The size of the protein peak was independent of the solution concentration. In addition to the protein, the solution in which the proteins are stored also contains buffers and surfactants that are added to improve the shelf life among other things. This can be seen in Figure 3D, where an additional peak is visible. This peak shifts to lower sizes with dilution, indicating soluble residuals in the protein suspension. These additive contamination coat the protein increasing the measured diameter relative to that observed in more dilute suspension. This slight shift of the protein peak position is apparent in Figure 3D for the more dilute suspension.

The relative number concentration of di- and trimers decreased when diluted as expected for all samples except for ubiquitin (A), a protein that was poorly suspended in the solution, leading to a higher multimer concentration. This may be caused by an extended waiting time between dispersion in the ultrasonic bath and measurement, or alternatively, too high protein concentration in the suspension might explain this size distribution. In addition to soluble residuals, non-soluble residuals, e.g. protein fragments, might agglomerate and yield additional peaks. To only see the monomer peak of a protein, a higher dilution is recommended. However, in this case we intended to see as many multimers as possible to be able to compare them to literature results, deliberately accepting additional artifact peaks such as contamination peaks or protein agglomerates.

In order to test the sizing accuracy in the diameter range above $20 \mathrm{~nm}$, we used polystyrene latex (PSL) particle size standards (with diameters of $73 \mathrm{~nm}$ and $107 \mathrm{~nm}$ ). These sizes are above the upper sizing limit of the Nano-DMA at $15 \mathrm{~L} / \mathrm{min}$ sheath flow. Therefore, for testing the $73 \mathrm{~nm}$ PSL, the sheath and aerosol flow rates were reduced to 4 and $0.4 \mathrm{~L} \mathrm{~min}^{-1}$, respectively, yielding a measurable diameter range of $4 \mathrm{~nm}$ to $138 \mathrm{~nm}$. For the $107 \mathrm{~nm}$ PSL the sheath and aerosol flow were further reduced to 2.51 and $0.25 \mathrm{~L} \mathrm{~min}^{-1}$, respectively, yielding a measurable size range from 
Table 1. Summary of the determined geometric mean diameter $D_{g}$ and their standard deviation (determined from 5 subsequent measurements), the corresponding literature or manufacturer reference value and the deviation from these. The trimer peaks of ubiquitin and myoglobin were clearly visible (as indicated in Figure 3) but due to the background peak of the solution it was not possible to determine the geometric mean diameter.

\begin{tabular}{lcccc}
\hline Substance & $\begin{array}{c}D_{g} \\
(\mathrm{~nm})\end{array}$ & $\begin{array}{c}\text { Standard Deviation } \\
(\mathrm{nm})\end{array}$ & $\begin{array}{c}\text { Literature Values } \\
(\mathrm{nm})\end{array}$ & $\begin{array}{c}\text { Deviation } \\
(\%)\end{array}$ \\
\hline Ubiquitin (Monomer) & 3.54 & \pm 0.004 & $3.6^{*}$ & 1.6 \\
Ubiquitin (Dimer) & 4.52 & \pm 0.008 & $4.5^{*}$ & 0.4 \\
Ubiquitin (Trimer) & - & - & $5.2^{*}$ & \\
Myoglobin (Monomer) & 4.53 & \pm 0.02 & $4.6^{*}$ & 1.5 \\
Myoglobin (Dimer) & 5.84 & \pm 0.07 & $5.7^{*}$ & 2.4 \\
Myoglobin (Trimer) & - & - & $6.45^{*}$ & \\
Albumin (Monomer) & 7.08 & \pm 0.01 & $7.1^{*}$ & 0.28 \\
Albumin (Dimer) & 9.07 & \pm 0.02 & $9.0^{*}$ & 0.7 \\
Ferritin & 13.57 & \pm 0.05 & 14.3 & 5.1 \\
PSL 73 nm & 73.6 & \pm 0.17 & 73 & 0.8 \\
PSL 107 nm & 109.2 & \pm 0.13 & 107 & 2.0 \\
\hline
\end{tabular}

*Bacher et al. 2001

$5 \mathrm{~nm}$ to $178 \mathrm{~nm}$.

For protein and PSL size distributions, we calculated the geometric mean diameter $D_{g} . D_{g}$ of the protein peaks was determined from the measured size distributions shown in Figure 3. Figure 4A shows the measured geometric mean diameter $D_{g}$ for all tested substances plotted against corresponding literature values (proteins) or certified diameters (PSL size standards). The different symbols represent the different substances. The 1:1 line (solid) is plotted to guide the eye, along with the dashed lines indicating $\pm 5 \%$ deviation. The repeatability of the sizing was determined from 5 repeated measurements. The standard deviation of $D_{g}$ was less than $1 \%$ (see Table 1), so we did not show error bars on Figure 4. The observed sizes confirm both previous studies (Kaufman, 1998; Bacher et al., 2001) and the manufacturer's data, and lie within 3\% (see Table 1), except for the protein ferritin $(5.1 \%)$. One explanation could be that the used ferritin material was already some years old and possibly decayed participial or was less stable. More details can also be found in Table 1.

In order to further test the reproducibility of the sizing, we operated two identical ECs (model 3082) equipped with a Nano-DMA (model 3085) in a tandem setup (Figure 2B). This time we used sucrose as the sample material. We selected four different diameters using one Nano-DMA and subsequently measured the size distribution with the second Nano-SMPS. In Figure 4B, the nominal diameter set at the first nano-DMA is plotted against $D_{g}$ measured by the Nano-SMPS. It follows from these measurements that the sizing accuracy of the Nano-DMA and the Nano-SMPS system is within $3 \%$.

\subsubsection{Performance of fast size distribution scans}

In the previous section we focused on quasi-stationary measurements. Here we will consider measurements with different scanning velocities. The tandem setup (Figure 2B) enables the investigation of very narrow, quasimonodisperse size distributions of known diameters. We used this capability to investigate the effect of different scan times/velocities $\left(3,5,10,20\right.$ and $50 \mathrm{~s}$ or $v_{s}=0.48,0.29,0.15,0.07$ and $0.03 \mathrm{~s}^{-1}$, respectively) on the sizing accuracy and potential smearing effects. To assess the performance under normal measurement scenarios, we acquired the size distribution over the maximum scan range, i.e., 2 to $65 \mathrm{~nm}$ with a $50 \mathrm{~s}$ scan. Due to the high sheath flow and the short scan time, the residence time in the Nano-DMA is rather short, limiting the scan range for faster scans. For 3 $\mathrm{s}$ the maximum scan range decreased to 2.21 to $60.4 \mathrm{~nm}$. The different scan ranges were considered in the scanning velocity $v_{s}$. An AIM scan cycle consists of an up scan where the DMA voltage is ramped up followed by a down-scan where the voltage is ramped down rapidly. Down-scan data are not used for the size distribution result. Up-scan and down-scan times can be chosen independently. The down-scan time for each scan was $5 \mathrm{~s}$, except for the $3 \mathrm{~s}$ up-scan measurement, where the down-scan time was set to $3 \mathrm{~s}$. We first selected particle sizes with a nominal diameter of (1) $33.4 \mathrm{~nm}$, (2) 14 and (3) $6.9 \mathrm{~nm}$. The resulting size distributions are shown in Figure 5A showing the averaged size distribution (5 subsequent scans) of $33.4 \mathrm{~nm}$ particles, 5B $(14 \mathrm{~nm})$ and $5 \mathrm{C}(6.9 \mathrm{~nm})$. The different colors and symbols 


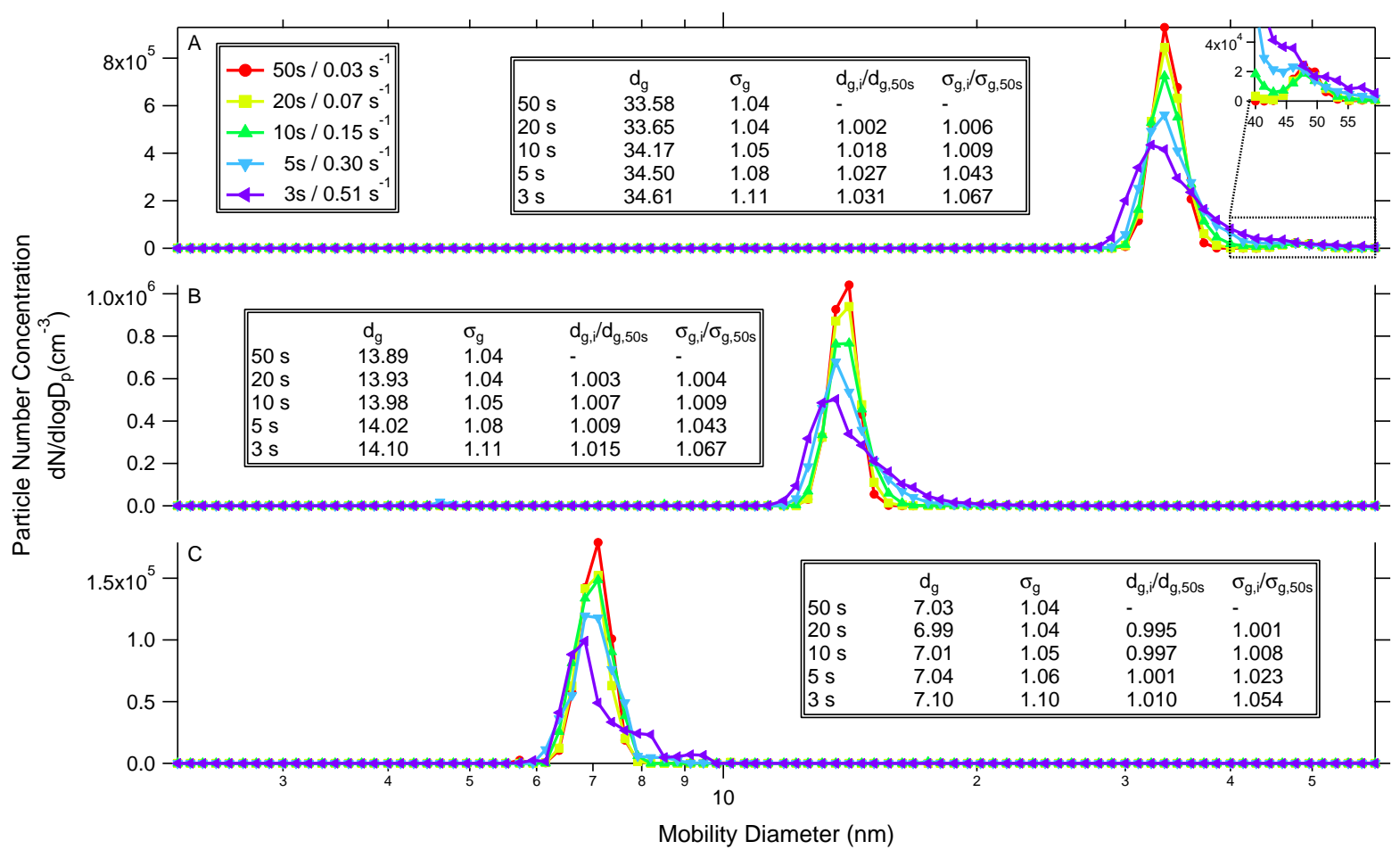

Figure 5. Fast scanning sucrose tests. Monodisperse size distributions of sucrose peaks using different scan times. Different sucrose solutions were used to generate the aerosol distribution. At the maximum of the distribution, the first Nano-DMA was used to get an even narrower distribution. Afterwards the scan time was varied to study the dependence of the mode diameter and width on it. The geometric mean diameter $D_{g}$ and geometric standard deviation $\sigma_{g}$ and their relative change compared to the reference scan of $50 \mathrm{~s}$ are also displayed. (Color figure available online.)

correspond to different scan times. The geometric mean diameter $D_{g}$ and geometric standard deviation $\sigma_{g}$ were calculated and are also displayed in Figure 5. Re-establishing the charge equilibrium in the X-ray bi-polar charger between the two Nano-DMAs leads to changes in the charging state of individual particles. Doubly-charged particles lose one charge, which make them appear at their true size in the second Nano-DMA, which is larger than the nominal diameter. This doubly charged peak can be seen in the small inset in Figure 5A at around $48 \mathrm{~nm}$. The recharged aerosol peak was neglected for the calculation of $D_{g}$ and $\sigma_{g}$ of the peak representing the singly charged particles. However, for the faster scan times, it was difficult for the size distribution displayed in Figure 5A to separate the doubly charged peak from the main peak. To retrieve the true $\sigma_{g}$, we fitted an exponentially modified Gaussian distribution through the measurements with 3,5 and $10 \mathrm{~s}$ scan time. For Figure 5B and C this approach was not necessary as the fraction of doubly charged particles in charge equilibrium was negligible for the considered sizes. For these scans the maximum possible scan range was chosen to allow for the fastest possible scan velocity of $0.48 \mathrm{~s}^{-1}$ at a scan time of $3 \mathrm{~s}$. The smearing effect at very fast scan times is clearly visible in the width and substantial asymmetry of the measured peak (Figure 5). The $\sigma_{g}$ of the peak increases from approximately 1.04 for the quasi-stationary measurement $(50 \mathrm{~s})$ to approximately 1.10-1.11 at the extremely short scan time of $3 \mathrm{~s}$. Still, $D_{g}$ is captured correctly to within $3 \%$ for all diameters. When the a scan velocity is reduced to $0.29 \mathrm{~s}^{-1}$ (= $5 \mathrm{~s}$ scan time across full size range), the smearing effect is much less. At $v_{s}=0.15 \mathrm{~s}^{-1}$ (= $10 \mathrm{~s}$ scan time) the smearing yields a change of less than $5 \%$ for $\sigma_{g}$. In conclusion, for all diameters and scan times, $D_{g}$ is accurate to within $3 \%$ for all scan times down to 3 s. Moreover $\sigma_{g}$ increases less than $7 \%$ for the different scan times compared to the reference measurement with a scan time of $50 \mathrm{~s}$. For scan times $>5 \mathrm{~s}$, both $D_{g}$ and $\sigma_{g}$ are reproduced to within $3 \%$. For a scan time of $20 \mathrm{~s}$, which corresponds to a scan velocity of $0.07 \mathrm{~s}^{-1}$, virtually no difference is visible compared to the $50 \mathrm{~s}$ scan. Thus, even a $20 \mathrm{~s}$ scan time yields results that are comparable to quasi-stationary measurements.

We tested the impact of smearing at short scan times for quasi-monodisperse size distributions. For the polydisperse size distributions typically encountered in field and lab experiments, the effects of smearing become smaller. 


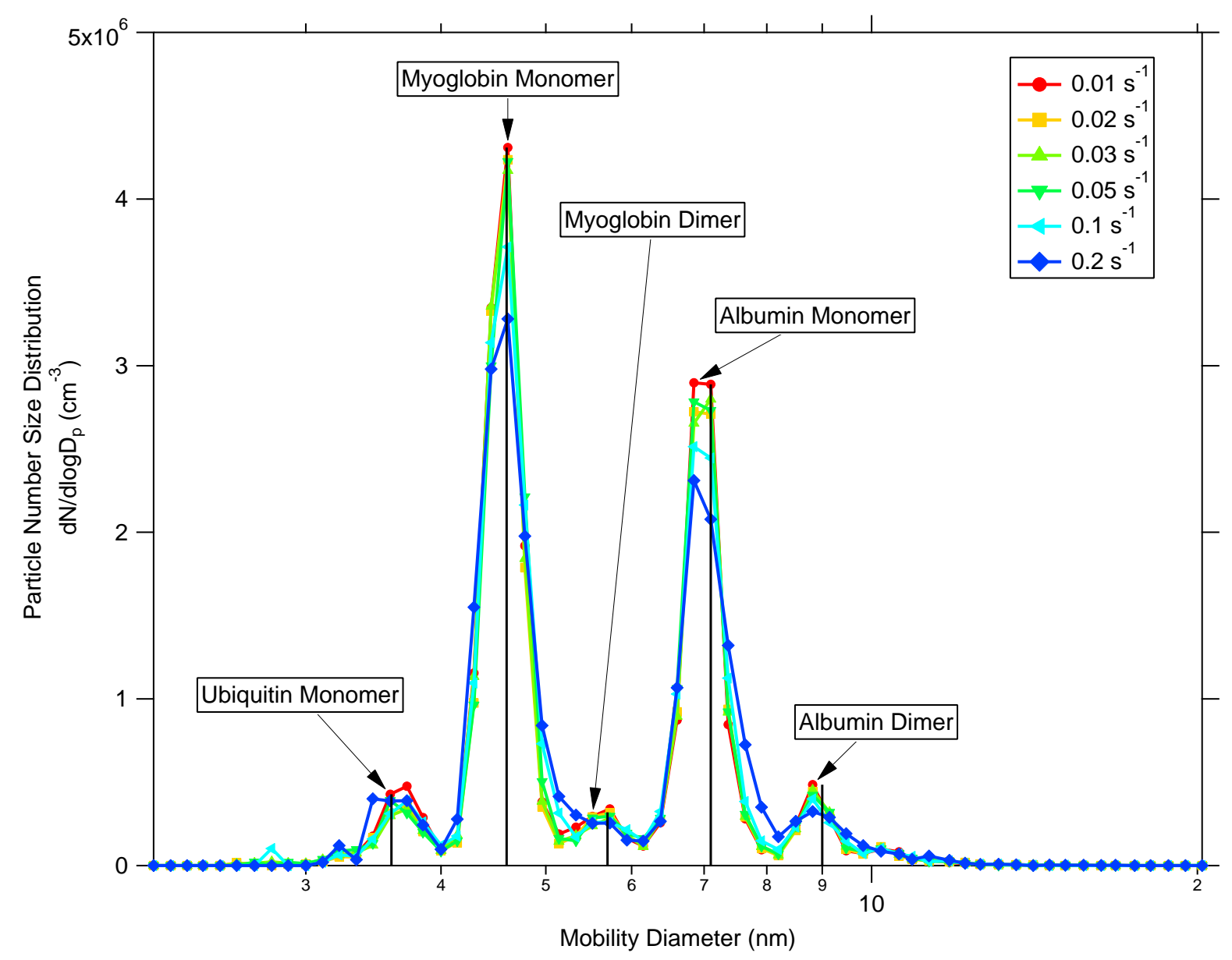

Figure 6. Fast scanning multi-peak solution of the proteins ubiquitin, myoglobin and albumin. Different scan times were tested to investigate their influence on sizing and resolution. The observed peaks correspond to: (1) ubiquitin monomer, (2) myoglobin monomer, (3) myoglobin dimer, (4) albumin monomer and (5) albumin dimer. (Color figure available online.)

Thus, even scan times $<20 \mathrm{~s}$ will produce comparable results to quasi-static scans. As previously noted by Wang et al. (2002) the delay time of the system must be determined very precisely to make the determination of $D_{g}$ independent of the scan velocity. Leaving the delay time uncorrected (delay time error of $0.15 \mathrm{~s}$ ) would yield a relative sizing error of $19 \%$ for a scan velocity of $0.48 s^{-1}$ (3 s scan). For a scan velocity of $0.03 s^{-1}$ (50 s scan), this relative sizing error decreases to $1 \%$. Thus, fast-scanning requires good calibration of the system beforehand. When this careful calibration is done, bias due to smearing effects decrease in importance; instead, counting statistics will often be the primary factor limiting the size resolution that can be achieved with very short scan times. Thus, for very low number concentrations measurements with longer scan times should be used or several short scans need to be averaged.

We further investigated the sizing accuracy and resolving power of the Nano-SMPS by examining a multi-peak size distribution for a scan range of one decade $(2-20 \mathrm{~nm})$, and different scanning velocities $\left(v_{s}=0.2,0.1,0.05\right.$, 0.03,0.02 and $\left.0.01 \mathrm{~s}^{-1}\right)$. We prepared a multi-protein solution consisting of ubiquitin, myoglobin and albumin. Using this multi-protein solution we observed a size distribution consisting of 5 peaks (see Figure 6 and diameters in table 1) corresponding to: (1) ubiquitin monomer, (2) myoglobin monomer, (3) myoglobin dimer (4) albumin monomer and (5) albumin dimer. Figure 6 compares single scan measurements of different scan velocities. Peaks corresponding to size differences of $\sim 1-2 \mathrm{~nm}$ are well resolved with a quasi-stationary measurement. Down to a scan velocity of $0.1 \mathrm{~s}^{-1}$, the size distributions can hardly be distinguished from quasi-stationary measurements. Smearing becomes visible for a scan velocity of $0.2 \mathrm{~s}^{-1}$. For this and higher scanning velocities, it becomes difficult to identify small peaks that sit 

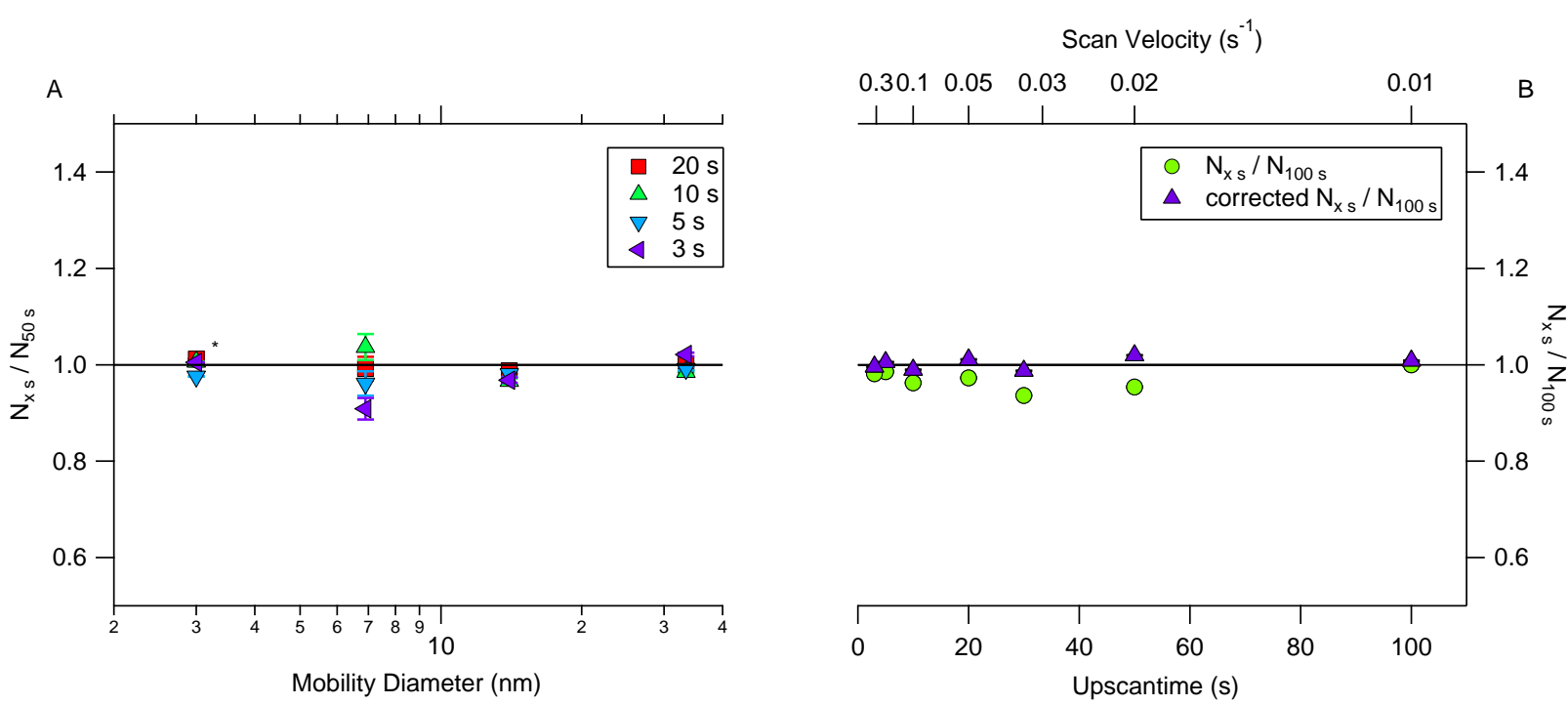

Figure 7. Reproducibility of integrated total number concentration for different diameters and scan times. (A) Setup B was used to investigate quasi-monodisperse size distributions (see Figure 5). The integrated number concentration was then compared to the reference scan (which had a scan time of $50 \mathrm{~s}$ ) plotting the ratio $N_{x \mathrm{~s}} / N_{50 \mathrm{~s}}$ against the measured diameter. For $3 \mathrm{~nm}\left({ }^{*}\right)$ it was not possible to use setup B, thus, the setup was changed to setup A. In this case the distribution is slightly broader. (B) Setup A was used to investigate the multi-peak distribution in Figure 6. The total number concentration was determined using different scan times and compared to the reference measurement using a scan time of $50 \mathrm{~s}$. (Color figure available online.)

in the tail of large peaks (e.g. peak 3).

The results obtained above can be directly compared to older SMPS systems. Collins et al. (2002) investigated the smearing caused by the TSI CPC 3010. They ramped a DMA from 10 to $10000 \mathrm{~V}$, which is very similar to our applied voltage range (10 to $9800 \mathrm{~V}$ ). Measurements of $300 \mathrm{~s}$ yielded quasi-stationary results. Already measurements with a $90 \mathrm{~s}$ scan time showed a significant smearing. For $30 \mathrm{~s}$ the peak concentration is only approximately $33 \%$ of the $300 \mathrm{~s}$ scan. In comparison, for the instrument tested here, the $3 \mathrm{~s}$ measurement still shows a peak concentration of approximately $50 \%$ compared to the reference scan.

\subsection{Reproducibility of number concentration measurement}

This section describes the precision with which the number concentration measurements can be made for fast size distribution scans. This is a second prerequisite, besides the sizing accuracy tested in the previous section, for precise size distribution measurements. For this purpose we used the size distribution measurements of quasi-monodisperse sucrose particles acquired with the tandem setup (Figure 2B). The first Nano-DMA was held at a fixed setpoint in order to select a quasi-monodisperse size-cut from a sucrose aerosol. The second Nano-SMPS scanned across the full size range with different scan times. The integrated particle number concentrations $\left(N_{x \mathrm{~s}}\right.$; where $\mathrm{x}$ is a placeholder for the scan duration) of the measured size distributions, such as those shown in Figure 5, were compared with $N_{50 \mathrm{~s}}$ measured with the quasi-stationary reference scan of $50 \mathrm{~s}$ duration.

We tested the following scan times/velocities: 3, 5, 10, 20 and 50 s corresponding to $v_{s}=0.48,0.29,0.15,0.07$ and $0.03 s^{-1}$, respectively. In Figure 7A the ratio of $N_{x \mathrm{~s}} / N_{50 \mathrm{~s}}$ is plotted against the selected diameters, whereby the particle size distribution was averaged over 5 measurements. The error bars correspond to the statistical uncertainty $\left(1 / C_{x \mathrm{~s}}+1 / C_{50 \mathrm{~s}}\right)^{1 / 2} \cdot N_{x \mathrm{~s}} / N_{50 \mathrm{~s}}$ with $C_{x \mathrm{~s}}$ being the total raw counts from 5 measurements for the corresponding scan time x s. The total number concentration is captured correctly for all scan times. Only for $7 \mathrm{~nm}$ aerosol particles did the total concentration deviate for different scan times (by 10\%). This scatter can be explained by lower counting statistics. At equilibrium, less than $1 \%$ of $7 \mathrm{~nm}$ aerosols (Wiedensohler, 1988) are singly charged, yielding very low particle concentrations after the DMA. Decreasing the scan time further decreases the counting statistics. Thus, for low concentration of small particles, scan times of at least $10 \mathrm{~s}$ or an average over several measurements are recommended. For this reason it was not possible to test $3 \mathrm{~nm}$ with the tandem-DMA setup. Only a very small 
A
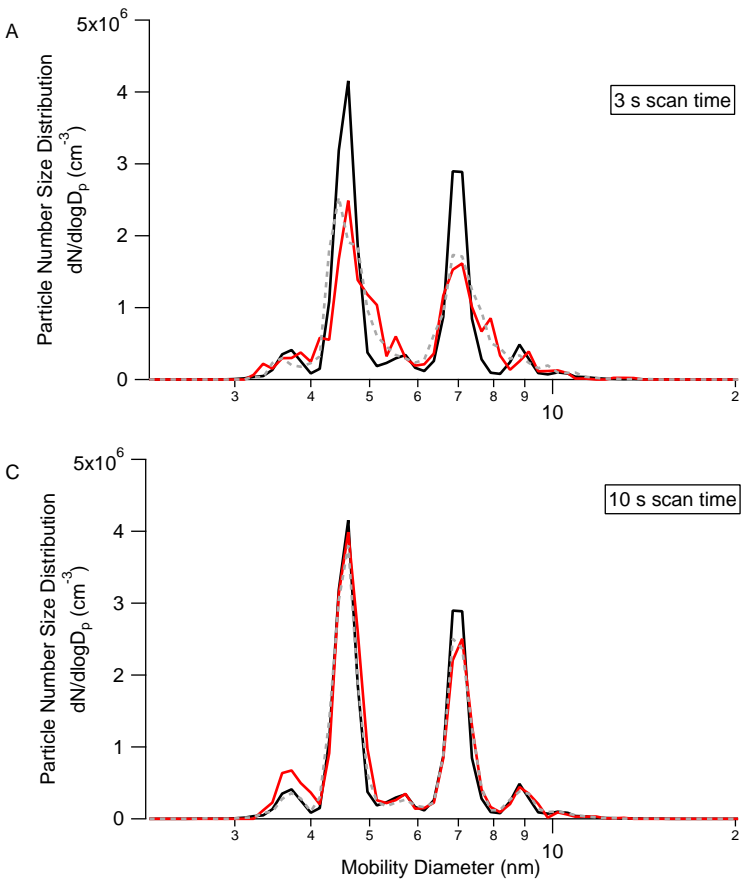
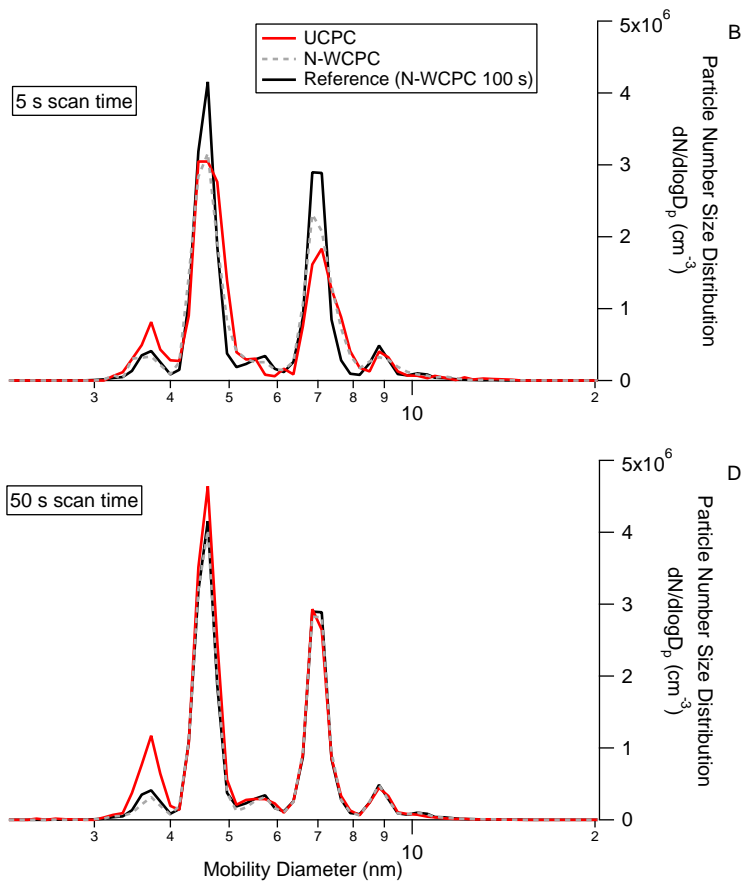

Figure 8. Comparison of the N-WCPC and the UCPC as detectors in the Nano-SMPS. The performance using different scan times of the N-WCPC and UCPC are compared to a reference scan of $100 \mathrm{~s}$ performed by the N-WCPC. (A) corresponds to $3 \mathrm{~s}$, (B) to $5 \mathrm{~s}$, (C) to $10 \mathrm{~s}$ and (D) to $50 \mathrm{~s}$. (Color figure available online.)

fraction of $3 \mathrm{~nm}$ particles is charged, so the fraction that penetrated through both Nano-DMAs was too low to be measured. Therefore, we directly connected the electrospray to the Nano-SMPS, yielding higher counts. Additionally, we decreased the scan range to $2-20 \mathrm{~nm}$. This additional measurement with a different setup is indicated with (*) in Figure 7A. The resulting distribution was slightly broader (true sucrose distribution with $\sigma_{g}=1.1$ ) and the total counts increased. This again improved the counting statistics significantly, thus, reducing the relative uncertainty of the $N_{x \mathrm{~s}}$ values. This test for $3 \mathrm{~nm}$ particles revealed a reproducibility of $\pm 3 \%$ for all scan times down to $3 \mathrm{~s}$.

We also tested the reproducibility of the total number concentration of a multiple quasi-monodisperse size distribution as a function of the scan time. Here we used the size distribution data from the protein mixture shown in Figure 6. Figure 7B shows the ratio of $N_{x \mathrm{~s}} / N_{100 \mathrm{~s}}$ in dependence on the scan time. Also here the error bars correspond to the statistical uncertainty $\left(1 / C_{x \mathrm{~s}}+1 / C_{100 \mathrm{~s}}\right)^{1 / 2} \cdot N_{x \mathrm{~s}} / N_{100 \mathrm{~s}}$.

The scans were performed in the following order: (1) $50 \mathrm{~s}\left(v_{s}=0.02 \mathrm{~s}^{-1}\right)$, (2) $30 \mathrm{~s}\left(0.03 \mathrm{~s}^{-1}\right)$, (3) $20 \mathrm{~s}(0.05$ $\left.\mathrm{s}^{-1}\right)$, (4) $10 \mathrm{~s}\left(0.1 \mathrm{~s}^{-1}\right),(5) 5 \mathrm{~s}\left(0.2 \mathrm{~s}^{-1}\right)$, (6) $3 \mathrm{~s}\left(0.3 \mathrm{~s}^{-1}\right)$ and (7) $100 \mathrm{~s}\left(0.01 \mathrm{~s}^{-1}\right)$. Even down to a scan time of $3 \mathrm{~s}$, the concentrations are comparable to the reference scan of $100 \mathrm{~s}$. The worse agreement of $50 \mathrm{~s}$ and $30 \mathrm{~s}$ compared to $3 \mathrm{~s}$ can be explained by a slight trend in the concentration of the generated aerosol from the electrospray. Thus, we added additional points to Figure $7 \mathrm{~B}$, correcting $N_{x \mathrm{~s}} / N_{100 \mathrm{~s}}$ for the drift in the electrospray. This test revealed a reproducibility of $\pm 2 \%$ for all scan times.

We demonstrated the reproducibility of the measured total number concentration using different scan times through the measurements described in this section, and have shown that fast scans can be just as precise as quasi-stationary scans. We did not test the accuracy of the measured total number concentration, as it is well known that uncertainties associated with the corrections for charging probability, diffusion losses and CPC counting efficiency are considerable in the sub-20 nm size range (Wiedensohler et al., 2012).

\subsection{Comparison UCPC versus $N$-WCPC}

We tested the well-established UCPC within the Nano-SMPS setup in comparison to the N-WCPC at different scan times (see Figure 1). Figure 8 shows the results: The solid black line corresponds to the reference single scan 
measured by the N-WCPC (scan time $100 \mathrm{~s}$ ). The dashed lines correspond to the N-WCPC single scans and the solid red lines to the UCPC single scans. The following scan velocities $v_{s}$ (scan times) were tested: (1) $0.3 s^{-1}(3 \mathrm{~s})$, (2) $0.2 s^{-1}(5 \mathrm{~s})$, (3) $0.1 s^{-1}(10 \mathrm{~s})$ and (4) $0.02 s^{-1}(50 \mathrm{~s})$. Both alternative setups were corrected for the system diffusion losses, single charge probability and the interference of doubly charged particles.

For sucrose particles, the two detectors have a very similar detection threshold. The cut-off diameter $D_{50}$, where $50 \%$ of the particles of a certain diameter are detected, is around $2.5 \mathrm{~nm}$ for both detectors. As the two detectors use different working liquids (water or butanol) their detection efficiency curves can depend on the chemical compositions of the detected particles. Due to the proteins' low water affinity, the UCPC has a better detection efficiency compared to the N-WCPC. The detection efficiency of ubiquitin is approximately three times higher for the UCPC compared to the N-WCPC (Kupc et al., 2013). To properly correct the measured multi-protein solution for the detection efficiency, it would be necessary to correct each peak separately. As we are only interested in the performance for fast scan times, we did not perform a CPC detection efficiency correction for this comparison. Thus, differences in the concentration directly reflect a higher counting efficiency. For the larger protein sizes there is hardly any difference in concentration, as the detection efficiency is not changed significantly.

The $100 \mathrm{~s}$ scan is shown as a quasi-stationary reference measurement that is not affected by smearing effects. For the $50 \mathrm{~s}$ scans, virtually no difference is visible compared to the reference scan (Figure 8D). The $10 \mathrm{~s}$ scan (Figure 8C) is virtually identical to the $100 \mathrm{~s}$ reference scan. For the $5 \mathrm{~s}$ (Figure 8B) and $3 \mathrm{~s}$ (Figure 8A) scans, differences to the $100 \mathrm{~s}$ scan become obvious. For these rapid scans the N-WCPC shows a slightly better performance. Due to the short scan time the counting statistics become poor, resulting in an increased noise. This is more pronounced for the UCPC due to the lower aerosol flow rate of only $50 \mathrm{~mL} \mathrm{~min}^{-1}$ compared to $300 \mathrm{~mL} \mathrm{~min}^{-1}$ in the N-WCPC. This is especially visible in Figure 8A, where the area of the ubiquitin peak is not captured correctly anymore. Often counting statistics are the limiting factor for size resolution rather than smearing. This is often pronounced in the sub- $20 \mathrm{~nm}$ diameter range, due to the low charging probability. Thus, for low aerosol concentrations and/or fast scanning the N-WCPC performs better.

\section{Conclusions}

During highly dynamic aerosol evolution like nucleation events, rapid growth rates of around $10 \mathrm{~nm} \mathrm{~h}^{-1}$ or more are observed in field experiments (Kulmala et al., 2004; Venzac et al., 2008). Lab experiments even show higher growth rates (Riccobono et al., 2012). One common method to estimate aerosol growth rates is to study the temporal evolution of the nucleation mode diameter (Lehtinen and Kulmala, 2003). With scan times of $60 \mathrm{~s}$ or more, the measurement will show a transient size distribution. Being able to reduce the scan time can, therefore, significantly enhance the quality of the analysis. Another possible application is the measurement of fresh emissions, such as seen during road side monitoring or direct emission measurements.

Here we present the results of the performance tests of the new TSI Nano-SMPS. Sizing accuracy and concentration precision have been investigated, as a function of the scan time and diameter. We showed that excellent results can be obtained at scan times down to $3 \mathrm{~s}$. The sizing accuracy of the new Nano-SMPS is within 3\% for several tested substances (proteins, sucrose and PSL reference particles) for all scan times. Due to the finite rise time of the CPC, decreasing the scan time causes a smearing of the size distribution. Some smearing effects are seen for scan velocities higher than $0.2 \mathrm{~s}^{-1}$ (this corresponds to approx. $7 \mathrm{~s}$ over the full size range), but the effect is small, resulting in a small reduction in the size resolving power. By decreasing the size range of the scan this smearing can be significantly decreased. In addition, the smearing will be less pronounced for polydisperse distributions, which are commonly observed in field and lab experiments.

The precision of the measured number concentration was also investigated for different scan times and aerosol distributions. The precision is within $\pm 3 \%$ for sufficient counting statistics. The variability can increase if the counting statistics are not sufficient. In applying this instrument to nucleation and growth experiments, low counting statistics might be a problem, especially for low nucleation and growth rates. However, for lab experiments where the formation rates of freshly nucleated particles can be rather high, this problem is not likely to occur. Also for vehicle emission measurements, depending on engine type, high particle concentrations are expected. The accuracy of the measured total number concentration, especially in the sub-20 nm size range, is limited by the uncertainties of the charging probability, diffusion losses and CPC counting efficiency. It also requires a well calibrated condensation particle 
counter as a reference. For these reasons, the determination of the accuracy in number remains a challenge for future studies.

The tested systems in general show neither sizing nor number concentration bias for fast scans. Very little smearing (corresponding to very little resolution loss) occurs for high scan velocities, i.e. short scan times without loss of accuracy or size resolution can be used. If counting statistics are insufficient for single scans, an average over multiple repetitions is an option. The delay time still needs to be determined properly to allow for accurate size determination. In addition decreasing the down scan time avoids loss of counting statistics compared to fewer but longer scans.

\section{Acknowledgments}

This work was supported by the EC Seventh Framework Programme (Marie Curie Initial Training Network "CLOUD-TRAIN" no. 316662) as well as the Swiss National Science Foundation. MG was funded by ERC-2013CoG 615922-BLACARAT.

\section{References}

Bacher, G., Szymanski, W. W., Kaufman, S. L., Zöllner, P., Blaas, D., Allmaier, G., 2001. Charge-reduced nano electrospray ionization combined with differential mobility analysis of peptides, proteins, glycoproteins, noncovalent protein complexes and viruses. Journal of Mass Spectrometry $36(9), 1038-1052$.

Birmili, W., Stratmann, F., Wiedensohler, A., Covert, D., Russell, L. M., Berg, O., 1997. Determination of differential mobility analyzer transfer functions using identical instruments in series. Aerosol Science and Technology 27, 215-223.

Chen, D.-R., Pui, D. Y., Hummes, D., Fissan, H., Quant, F., Sem, G., 1998. Design and evaluation of a nanometer aerosol differential mobility analyzer (Nano-DMA). Journal of Aerosol Science 29 (5), 497-509.

Chen, D.-R., Pui, D. Y., Kaufman, S. L., 1995. Electrospraying of conducting liquids for monodisperse aerosol generation in the $4 \mathrm{~nm}$ to $1.8 \mu \mathrm{m}$ diameter range. Journal of Aerosol Science 26 (6), 963-977.

Collins, D. R., Flagan, R. C., Seinfeld, J. H., 2002. Improved inversion of scanning DMA data. Aerosol Science \& Technology 36 (1), 1-9.

Hering, S. V., Stolzenburg, M. R., 2005. A method for particle size amplification by water condensation in a laminar, thermally diffusive flow. Aerosol Science \& Technology 39 (5), 428-436.

Hering, S. V., Stolzenburg, M. R., Quant, F. R., Oberreit, D. R., Keady, P. B., 2005. A laminar-flow, water-based condensation particle counter (WCPC). Aerosol Science \& Technology 39 (7), 659-672.

Hoet, P. H., Brüske-Hohlfeld, I., Salata, O. V., 2004. Nanoparticles-known and unknown health risks. Journal of Nanobiotechnology 2 (1), 12.

Johnson, T., Caldow, R., Poecher, A., Mirme, A., Kittelson, D., 2004. A new electrical mobility particle sizer spectrometer for engine exhaust particle measurements. Tech. rep., SAE Technical Paper.

Kaufman, S. L., 1998. Analysis of biomolecules using electrospray and nanoparticle methods: the gas-phase electrophoretic mobility molecular analyzer (GEMMA). Journal of Aerosol Science 29 (5), 537-552.

Kaufman, S. L., 2000. Electrospray diagnostics performed by using sucrose and proteins in the gas-phase electrophoretic mobility molecular analyzer (GEMMA). Analytica chimica acta 406 (1), 3-10.

Knobel, L., Weinhold, H., Gandhi, J., Wiedensohler, A., H-J, S., 2013. Application of a X-ray charger for SMPS measurements. Proceedings to European Aerosol Conference (Prague).

Knutson, E., Whitby, K., 1975. Aerosol classification by electric mobility: apparatus, theory, and applications. Journal of Aerosol Science 6 (6), $443-451$.

Kulmala, M., Laakso, L., Lehtinen, K. E. J., Riipinen, I., Dal Maso, M., Anttila, T., Kerminen, V. M., Horrak, U., Vana, M., Tammet, H., 2004. Initial steps of aerosol growth. Atmospheric Chemistry and Physics 4 (11/12), 2553-2560.

Kulmala, M., Petaja, T., Nieminen, T., Sipilä, M., Manninen, H. E., Lehtipalo, K., Dal Maso, M., Aalto, P. P., Junninen, H., Paasonen, P., Riipinen, I., Lehtinen, K. E., Laaksonen, A., Kerminen, V. M., 2012. Measurement of the nucleation of atmospheric aerosol particles. Nature Protocols 7 (9), 1651-67.

Kupc, A., Bischof, O., Tritscher, T., Beeston, M., Krinke, T., Wagner, P. E., 2013. Laboratory characterization of a new nano-water-based CPC 3788 and performance comparison to an ultrafine butanol-based CPC 3776. Aerosol Science \& Technology 47 (2), $183-191$.

Lehtinen, K., Kulmala, M., 2003. A model for particle formation and growth in the atmosphere with molecular resolution in size. Atmospheric Chemistry and Physics 3 (1), 251-257.

Lòpez-Yglesias, X., Flagan, R. C., 2013. Population balances of micron-sized aerosols in a bipolar ion environment. Aerosol Science \& Technology $47(6), 681-687$

Mamakos, A., Ntziachristos, L., Samaras, Z., 2008. Differential mobility analyser transfer functions in scanning mode. Journal of Aerosol Science 39 (3), 227-243

Merikanto, J., Spracklen, D., Mann, G., Pickering, S., Carslaw, K., 2009. Impact of nucleation on global CCN. Atmospheric Chemistry and Physics $9(21), 8601-8616$.

Olfert, J. S., Kulkarni, P., Wang, J., 2008. Measuring aerosol size distributions with the fast integrated mobility spectrometer. Journal of Aerosol Science 39 (11), 940-956.

Peters, A., Wichmann, H. E., Tuch, T., Heinrich, J., Heyder, J., 1997. Respiratory effects are associated with the number of ultrafine particles. American Journal of Respiratory and Critical Care Medicine 155 (4), 1376-1383. 
Quant, F., Caldow, R., Sem, G., Addison, T., 1992. Performance of condensation particle counters with three continuous-flow designs. Journal of Aerosol Science 23, 405-408.

Reineking, A., Porstendörfer, J., 1986. Measurements of particle loss functions in a differential mobility analyzer (TSI, model 3071) for different flow rates. Aerosol Science and Technology 5 (4), 483-486.

Riccobono, F., Rondo, L., Sipilä, M., Barmet, P., Curtius, J., Dommen, J., Ehn, M., Ehrhart, S., Kulmala, M., Kürten, A., et al., 2012. Contribution of sulfuric acid and oxidized organic compounds to particle formation and growth. Atmospheric Chemistry and Physics 12 (20), 9427-9439.

Riccobono, F., Schobesberger, S., Scott, C. E., Dommen, J., Ortega, I. K., Rondo, L., Almeida, J., Amorim, A., Bianchi, F., Breitenlechner, M., et al., 2014. Oxidation products of biogenic emissions contribute to nucleation of atmospheric particles. Science 344 (6185), 717-721.

Russell, L. M., Flagan, R. C., Seinfeld, J. H., 1995. Asymmetric instrument response resulting from mixing effects in accelerated DMA-CPC measurements. Aerosol Science \& Technology 23 (4), 491-509.

Shah, S. D., Cocker, D. R., 2005. A fast scanning mobility particle spectrometer for monitoring transient particle size distributions. Aerosol Science \& Technology 39 (6), 519-526.

Stocker, T. F., Dahe, Q., Plattner, G.-K., 2013. Climate Change 2013: The Physical Science Basis. Working Group I Contribution to the Fifth Assessment Report of the Intergovernmental Panel on Climate Change. Summary for Policymakers (IPCC, 2013).

Tiede, K., Boxall, A. B., Tear, S. P., Lewis, J., David, H., Hassellöv, M., 2008. Detection and characterization of engineered nanoparticles in food and the environment. Food Additives and Contaminants 25 (7), 795-821.

Venzac, H., Sellegri, K., Laj, P., Villani, P., Bonasoni, P., Marinoni, A., Cristofanelli, P., Calzolari, F., Fuzzi, S., Decesari, S., et al., 2008. High frequency new particle formation in the Himalayas. Proceedings of the National Academy of Sciences 105 (41), 15666-15671.

Wang, J., McNeill, V. F., Collins, D. R., Flagan, R. C., 2002. Fast mixing condensation nucleus counter: application to rapid scanning differential mobility analyzer measurements. Aerosol Science \& Technology 36 (6), 678-689.

Wang, S. C., Flagan, R. C., 1990. Scanning electrical mobility spectrometer. Aerosol Science \& Technology 13 (2), $230-240$.

Weingartner, E., Gysel, M., Baltensperger, U., 2002. Hygroscopicity of aerosol particles at low temperatures. 1. new low-temperature H-TDMA instrument: Setup and first applications. Environmental Science \& Technology 36 (1), 55-62.

Wiedensohler, A., 1988. An approximation of the bipolar charge distribution for particles in the submicron size range. Journal of Aerosol Science 19 (3), 387-389.

Wiedensohler, A., Birmili, W., Nowak, A., Sonntag, A., Weinhold, K., Merkel, M., Wehner, B., Tuch, T., Pfeifer, S., Fiebig, M., et al., 2012. Mobility particle size spectrometers: harmonization of technical standards and data structure to facilitate high quality long-term observations of atmospheric particle number size distributions. Atmospheric Measurement Techniques 5, 657-685.

Zhu, Y., Hinds, W. C., Kim, S., Shen, S., Sioutas, C., 2002. Study of ultrafine particles near a major highway with heavy-duty diesel traffic. Atmospheric Environment 36 (27), 4323-4335. 